\title{
Metastatic solitary vaginal nodule from a primary rectal tumor.
}

\section{Fade Alawneh}

King Hussein Cancer Center

Mohamad K. Abou Chaar ( $\nabla$ ma.12564@khcc.jo )

King Hussein Cancer Center https://orcid.org/0000-0002-9584-2184

Lara Rabah

King Hussein Cancer Center

Qais Shatnawi

King Hussein Cancer Center

Azza Gharaibeh

King Hussein Cancer Center

Omar Jaber

King Hussein Cancer Center

Faiez Daoud

King Hussein Cancer Center

\section{Case report}

Keywords: colorectal cancer, vaginal metastasis, adenocarcinoma

Posted Date: August 6th, 2020

DOI: https://doi.org/10.21203/rs.3.rs-53505/v1

License: (c) (1) This work is licensed under a Creative Commons Attribution 4.0 International License.

Read Full License 


\section{Abstract}

Introduction: An isolated vaginal metastasis from a primary colorectal adenocarcinoma is extremely rare. Based on meticulous literature review, only seven cases were reported from 1956 - 2013.

Case Presentation: We present a case of a 52-year-old female patient diagnosed with rectal adenocarcinoma, underwent low anterior resection of the primary tumor, and then was found to have an asymptomatic isolated vaginal metastasis that was completely excised.

Conclusion: Isolated vaginal metastatic disease is one of the rarest entities in surgical oncology with only limited cases being reported in the literature. A diagnosis can be challenging if the metastatic lesion is small and asymptomatic even with the most advanced radiological assessment tools. A meticulous examination should be done pre-operatively in order to rule out such diagnosis.

\section{Introduction}

Primary vaginal neoplasm is one of the rarely encountered malignancies, with 17,600 cases reported worldwide in 2018 [1]. Vaginal metastatic lesions are extremely rare, with only 51 cases reported to date, and only seven of which reported isolated vaginal metastasis [2-4].

\section{Case Presentation}

Our patient is a 52-year-old female, known to have hepatitis B, married with six children with a single history of cesarean delivery, and non-smoker. In March 2017, she started to notice weight loss, loss of appetite, and alteration in bowel habits associated with minimal bleeding per rectum. A colonoscopy was ordered by her primary care physician, which showed a mass at $12 \mathrm{~cm}$ from the anal verge. Eight months later, she was referred to King Hussein Cancer Center (KHCC) for further management. Initial laboratory

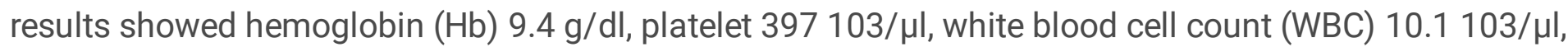
creatinine $0.7 \mathrm{mg} / \mathrm{dl}$, and carcinoembryonic antigen level $5.36 \mathrm{ng} / \mathrm{ml}$. Her physical examination showed a soft lax abdomen without any lymphadenopathy, and upon digital rectal examination (DRE) a mass $7 \mathrm{~cm}$ from the anal verge was palpated. Magnetic resonance imaging (MRI) study showed circumferential wall thickening of the upper rectum, $13.5 \mathrm{~cm}$ proximal to the anal verge, the involved segment measured approximately $7.0 \mathrm{~cm}$ in length, with severe stranding of perirectal fat and possible infiltration of the anterior aspect of the mesorectal fascia. In addition to multiple enlarged lymph nodes within the perirectal and presacral spaces, and along the course of the right internal iliac vessels (Fig. 1). Computed tomography (CT) whole-body scan was negative for metastatic disease. After counseling the patient and her family, a multidisciplinary clinic (MDC) decision was to go for low anterior resection, being staged T3N2. Under general anesthesia and via a lower abdominal vertical incision, the peritoneal cavity was reached, and meticulous search for metastasis in the liver, mesentery, omentum, and peritoneal surfaces was negative. The sigmoid colon was dissected from its lateral peritoneal attachment, followed by ligation of the inferior mesenteric artery and preservation of the left colic artery. The tumor was found to 
be adherent to the posterior vaginal wall, which necessitated gentle shaving of the tumor without violating the vaginal entity. Colo-rectal end-to-end primary anastomosis via gastro-intestinal endostapler (GIE) was performed; hemeostasis was secured, followed by drain insertion, closure, and dressing. The patient was monitored in the intermediate care unit (IMU) for 24 hours postoperatively, and then was transferred to the surgical ward. On postoperative day 3 , the patient reported passage of gas from the vagina, and with further questioning; she admitted to having this complaint even before the surgery.

Bedside speculum examination revealed a fungating mass $2 \times 3 \mathrm{~cm}$ in the middle of the posterior vaginal wall, $4 \mathrm{~cm}$ from the introitus, not limited to the vaginal mucosa. A biopsy was taken, and it came back with a diagnosis of metastatic colonic adenocarcinoma, based on the morphology and immunohistochemical stains (positive staining for CDX2 and negative staining for PAX8), (Fig. 2). The patient was discharged home without any complications. Final histopathological analysis of the resected specimen confirmed the diagnosis of moderately differentiated adenocarcinoma, stating that the tumor is $2 \mathrm{~mm}$ away from the anterior circumferential margin involvement and with negative resection margins (RO). After two months, the patient followed up with the gynecology clinic at KHCC, where she was reevaluated and informed about the need to perform a wide local excision of the posterior vaginal wall with a potential risk of anal sphincter injury. In late February of 2018, the patient underwent wide local excision of the posterior vaginal wall with primary repair, approximately a $2.5 \times 1.5 \mathrm{~cm}$ lesion was completely excised. The patient was discharged home the following day without any complications, and after one month, was started on adjuvant chemotherapy (10 cycles of Oxaliplatin, Leucovorin, and 5-Fluorouracil) and radiotherapy ( 25 fractions, 5000 cGy). The patient continued with regular follow-up at our clinics, and her most recent imaging studies, two years postoperatively, showed complete disease remission and no complications.

\section{Discussion And Conclusion}

Primary vaginal cancer is rare and represents only $1-2 \%$ of all gynecological malignancies [5].

Squamous cell carcinoma accounts for $90 \%$ of confirmed cases and only $8 \%$ are adenocarcinomas, most of which are secondary to uterine, rectal, ovarian, breast, pancreatic, or renal tumor [6,7]. Metastasis of colorectal carcinoma to the female genital tract is usually to the ovaries followed by the vagina and endometrium [8], knowing that the most common site for colorectal cancer metastasis is the liver [9]. Some of the suggested hypotheses for colorectal vaginal metastasis formation are; a) direct infiltration via the Pouch of Douglas, b) lymphovascular pathways, c) fallopian tubes pathway $[7,10]$. Whitelaw et al reported the first case of metastatic adenocarcinoma to the lateral wall of the vagina and the urethra. The patient presented at that time with vaginal spotting, status-post supravaginal hysterectomy, and was later found to have a primary sigmoid cancer, all of which were excised surgically with adjuvant radium therapy but unfortunately; the patient died 39 months after the onset of symptoms [11]. In 1966, Raider reported the first case of isolated anterior vaginal metastasis in a 63-year-old woman who underwent left colectomy for an obstructing mass, and after two years, she complained of vaginal bleeding that revealed a lesion. She was primarily treated with nitrogen mustard, followed by cobalt and radium mold therapy due to recurrence. The patient then developed an ileovaginal fistula that necessitated surgical excision 
after which she was in remission [12]. A review done by $\mathrm{Ng}$ and Aly in 2013 reported that the majority of vaginal metastasis cases had vaginal bleeding, followed by discharge, staining, heaviness, and perianal discomfort. They emphasized that due to the lack of reporting, a definitive treatment plan is still not present. Multiple studies reported different modalities from local treatment with radiotherapy to a more aggressive approach, including hysterectomy and bilateral salpingo-oophorectomy, according to the extent of the disease [13]. To the best of our knowledge, this is the first case to report gas passage sensation with an intact vaginal wall revealing metastatic nodule. It is worth mentioning that vaginal symptoms can be the first presentation for patients with metastatic colorectal carcinoma [7, 11, 14], and clinical examination remains superior to any other imaging modality [3].

\section{Abbreviations}

KHCC

King Hussein Cancer Center

$\mathrm{Hb}$

Hemoglobin

WBC

White blood cell

DRE

Digital rectal examination

MRI

Magnetic resonance imaging

CT

Computed tomography

MDC

Multidisciplinary clinic

GIE

Gastro-intestinal endostapler

IMU

Intermediate care unit

\section{Declarations}

\section{Ethics approval and consent to participate}

NA

\section{Consent for publication}


We have obtained written informed consent from the patients to publish this report.

\section{Availability of data and materials}

NA

\section{Competing interests}

NA

\section{Funding}

NA

\section{Authors' contributions}

MK and LR performed the literature search and wrote the manuscript guided by FA. OJ obtained and reviewed the pathology samples and provided the images. AG reviewed the case and provided radiological images. FA, QS and FD oversaw the manuscript's inception, guided the literature search, counseled the patient, performed the procedures, wrote portions of the manuscript, and provided major revisions. All authors read and approved the final manuscript.

\section{Acknowledgments}

Not applicable.

\section{References}

1. Torre LA, Bray F, Siegel RL, Ferlay J, Lortet-Tieulent J, Jemal A. Global cancer statistics, 2012. Cancer J Clin. 2015;65(2):87-108.

2. Ng HJ, Aly EH. Vaginal metastases from colorectal cancer. Int J Surg. 2013;11(10):1048-55.

3. D'Arco F, Pizzuti LM, Romano F, Natella V, Laccetti E, Storto G, ... Mainenti PP. MRI findings of a remote and isolated vaginal metastasis revealing an adenocarcinoma of the mid-sigmoid colon. Polish journal of radiology. 2014;79:33.

4. Yasuyama A, Noura S, Matsumura T, Hirota M, Takada A, Koga C, ... Hasegawa J. A Resected Case of the Vaginal Metastasis from Rectal Cancer. Gan to kagaku ryoho. Cancer chemotherapy. 2017;44(12):1434-6. 
5. Di Donato V, Bellati F, Fischetti M, Plotti F, Perniola G, Panici PB. Vaginal cancer. Crit Rev Oncol Hematol. 2012;81(3):286-95.

6. Yagci G, Cetiner S, Dede M, et al. True vaginal metastasis of rectal cancer. Indian J Surg. 2005;67:270-72.

7. Marchal F, Leroux A, Hoffstetter S, et al. Vaginal metastasis revealing colon adenocarcinoma. Int $J$ Colorectal Dis. 2006;21:861-62.

8. Mazur MT, Hsueh S, Gersell DJ. Metastases to the female tract. Analysis of 325 cases. 1984;53:1978e84.

9. Fong Y, Cohen AM, Fortner JG, Enker WE, Turnbull AD, Coit DG, ... Brennan MF. Liver resection for colorectal metastases. J Clin Oncol. 1997;15(3):938-46.

10. Raider L. Remote vaginal metastases from carcinoma of the colon. Am J Roentgenol Radium Ther Nucl Med. 1966;97(4):944e50.

11. Whitelaw GP, Leard SE, Parsons L, Sherwin RP. Carcinoma of the large bowel with metastasis to the genitalia: Report of two cases. AMA archives of surgery. 1956;73(1):171-8.

12. RAIDER L. Remote vaginal metastases from carcinoma of the colon. Am J Roentgenol. 1966;97(4):944-50.

13. Ng HJ, Aly EH. Vaginal metastases from colorectal cancer. Int J Surg. 2013;11(10):1048-55.

14. Ceccaroni M, Paglia A, Ruffo G, Scioscia M, Bruni F, Pesci A, Minelli L. Symptomatic vaginal bleeding in a postmenopausal woman revealing colon adenocarcinoma metastasizing exclusively to the vagina. Journal of Minimally Invasive Gynecology. 2010;17(6):779-81.

\section{Figures}



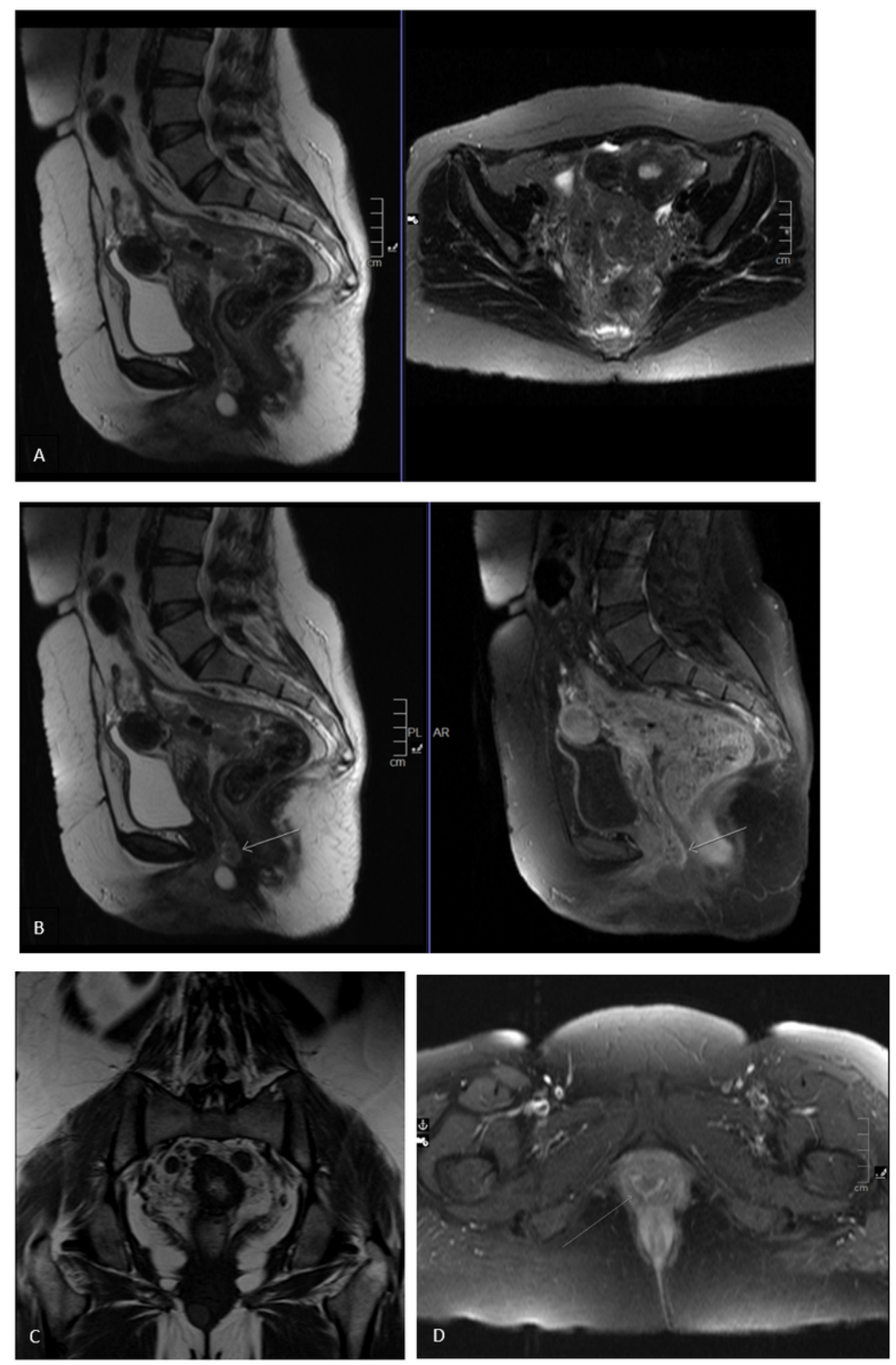

\section{Figure 1}

Locally advanced high rectal tumor. A) Sagittal T2 weighted MR image shows concentric high rectal tumor. Axial STIR weighted images shows extensive infiltration into mesorectal fat. B) Coronal T1 weighted MR image shows the rectal tumor with infiltration into the mesorectal fat. Multiple enlarged mesorectal lymph nodes. C) Sagittal T2 weighted MR image shows irregular mass of intermediate to high signal intensity involving the posterior wall of the lower vagina. On sagittal T1 post-contrast there is mild 
heterogeneous enhancement of the lesions. D) Axial T1 post contrast weighted MR image shows the small lesion in the posterior wall of the lower vagina.
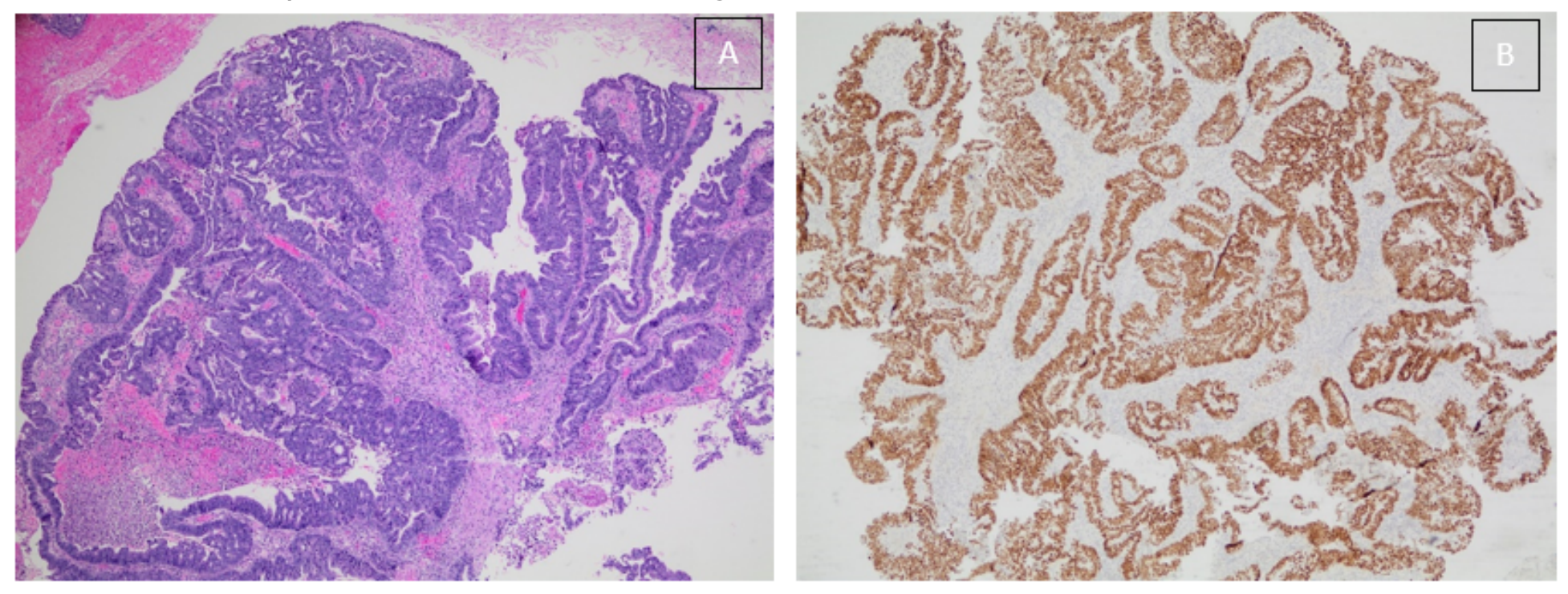

\section{Figure 2}

Microscopic examination of the tumor. A) Metastatic colonic adenocarcinoma to vaginal wall. Hematoxylin and eosin stain, 40X. B) CDX2 positive immunohistochemical stain in metastatic colonic adenocarcinoma to vaginal wall. CDX2 immunohistochemical stain, 40X. 